Research letter

\title{
Extended application of dose-response models of infection on injectable insulin products in vials
}

\author{
Mostafa Essam Eissa
}

HIKMA Pharma Pharmaceutical Company, Giza, Egypt

Received 13 March 2016, Revised 14 April 2016, Accepted 18 May 2016

(C) 2016, Eissa M.E.

(c) 2016, Russian Open Medical Journal

Abstract: Microbial contamination from in-use application of multidose pharmaceutical products constitutes health hazard to the consumer especially when considering injectable medicines. In the present study, an attempt has been made to integrate dose-response models of infections with pharmacopeial preservative efficacy test (PET) in a single simulation study that supposed accidental reuse of hypodermic syringe. Probability risk ratio of infection from assumed equal doses of two types of microbes by two routes of infection was greater than 1300 at most equivalent administration doses when considering the same reduction factor for both bacteria in the antimicrobial efficacy test (AET). The probability of infection would diminish much more rapidly for Staphylococcus aureus rather than Pseudomonas aeruginosa. The short interval of product administration increased the risk of infection with regular-type of Insulin vials. The current quantitative study provided extension and made the best use of the standard AET but also highlighted its limitations.

Keywords: preservative efficacy test, hypodermic syringe, Pseudomonas aeruginosa, Staphylococcus aureus, regular-type of insulin

Cite as Eissa ME. Extended application of dose-response models of infection on injectable insulin products in vials. Russian Open Medical Journal 2016; 5 : e0305.

Correspondence to Mostafa Essam Eissa. Adddress: P.O. Box 1913, Plot no.1, $6^{\text {th }}$ of October City, Giza, Egypt. Phone: 00201006154853.

E-mail: mostafaessameissa@yahoo.com

\section{Introduction}

Microbial intrusions emerging from crude materials and amid processing, drugs might get to be defiled amid storage and during consumption. Sorts and levels of "in-use" tainting are practically difficult to anticipate and just constrained distributed information is obtainable. For medications that are brought into ordinarily sterile parts of the body, the potential hazards are significant. A survey of microbial tainting of intravenous (IV) liquids amid use proposed that the little quantities of mostly non-pathogenic microbes were by and large all around endured and that disease was an uncommon event [1].

While microbial pollution of sterile items amid production processes is currently seldom an issue, microbial invasion of IV liquids and device amid clinical use is still known to happen. From a writing review (1971-1983), Denyer (1984) presumed that the microbial contamination of IV liquids from packaged bottles and collapsible plastic dosage forms during consumption was of the magnitude of 4.3 and $2.6 \%$, in order; by and large, the observed bioburden was available in low numbers [1]. When the aseptic product integrity is broken, the microbial infection risk with its associated produced toxins is largely dependent on the ability of the product formulation to support microbial proliferation [2]. In assessing the outcome of the screening of microbial contamination during product consumption, it must be understood that pollution levels in utilized items reflect the bioburden input from the patient as well as the survival capabilities of the contaminant in the medicinal drug. Unfortunately, Koerner et al. 1997 reported a sudden dissemination of septicemia which was accounted for in an intense cardiology ward. The flare-up was credited to Enterobacter cloacae (member of Enterobacteriaceae) intrusion of heparin mixtures that were mixed in non-clinical places abutting the ward from packs of IV liquids, which stayed open for 24 hours or even more at room temperature, and non-single use heparin vials [3].

The above described challenges stimulated the study of risk and probability of infection that could arise from contaminated multidose injectable products, using commercial Insulin vials that are commonly found in the drug market. As diabetes is considered one of the most commonly observed frequently chronic diseases in the world, the aim of the current research is to combine preservative efficacy test (PET) with dose-response models of infection using indicator microbes in simulation study that integrate the most critical parameters which influence the infectivity of the contaminated pharmaceutical with the possible hazard to the health of patients.

\section{Material and Methods}

\section{PET design and method}

PET test was performed for each product type (nine different forms) five times. Product categories included three varieties with three subset types. The test parameters and guideline references were done as demonstrated by Eissa and Mahmoud (2015) [4, 5]. The outcome of the five treatments were combined and averaged. 
Table 1. Dose-response infection model parameters per route of administration using critical or indicator microorganisms

\begin{tabular}{|c|c|c|c|c|c|c|}
\hline Agent & Best fit model & Optimized parameter(s) & LD50/ID50 & Route & Dose units & Refs. \\
\hline Staphylococcus aureus: Dose response model & exponential & $\mathrm{k}=7.64 \times 10^{-8}$ & $9.08 \times 10^{6}$ & subcutaneous & $\mathrm{CFU} / \mathrm{cm}^{2}$ & {$[6]$} \\
\hline Pseudomonas aeruginosa (bacterimia): Dose response model & exponential & $\mathrm{k}=1.05 \times 10^{-4}$ & $6.61 \times 10^{3}$ & injected in eyelids & CFU & [7] \\
\hline
\end{tabular}

Table 2. Active pharmaceutical ingredient (API) and other additives that may enhance or modify the preservation power of the formulae as in the instruction pamphlet of the manufactured products

$\begin{array}{lcc}\text { Type of component } & \text { Regular, Neutral and Soluble (RNS) } & \text { Insulin product type } \\ \text { API } & \text { Soluble crystals of zinc-Insulin } & \begin{array}{c}\text { Suspension-forming crystalline insulin } \\ \text { with zinc and protamine } \\ \text { Inactive Components }\end{array} \\ \text { pH range } & \text { Glycerol and WFI } & \text { Glycerol, sodium phosphate and WFI } \\ \text { Glycerol, sodium phosphate, protamine } \\ \text { sulphate, zinc oxide and WFI } \\ 6.9-7.5\end{array}$

WFI, water for injection.

\section{Principle theory}

Reference or indicator microorganisms and parameters of dose-response models of infections with references were presented in Table 1. On the other hand, Table 2 shows the active pharmaceutical ingredient (API) and other inactive components that may influence the antimicrobial strength of the three main categories of Insulin product vial forms. Preservatives and the usual dosing regimen used for each formula type are indicated in Table 3 as per manufacturer leaflet. Importantly, Table 4 gathers all parameters criteria for assessing the risk of infection. Assumptions about modeling of dose-response of infection to complement antimicrobial efficacy test (AET) were as the following:

1 - The maximum risk analysis using worst case scenario was used to measure the highest possibility of infection hazard at which the users of the multidose medicinal dosage forms are exposed, i.e. assuming high contamination level and using the lowest logarithmic reduction (LR) values as cut-off figures even if no microbial populations were recovered from AET after seven days.

2 - The current study assumed accidental reuse of the same hypodermic syringe. The needle in such case presented the medium for transfer of skin flora to the vial and vice versa. However, the present model supposed to use single contamination spot simulation, while multiple spots contamination will be addressed in separate evaluation experiment. The transfer factor would be calculated as $100 \%$ from the dermis to the needle surface according to the surface area of the needle as a maximum potential risk.

3 - Based on the common Insulin administration regimes two common possible routes for infections were used in this simulation study as determined in Table 1. An exponential model of infection would relate microbial reduction from AET, dosing administration for each type of Insulin and assumed level of indicator microorganisms in colony forming unit (CFU) with probability of infection that was expressed as percentage.

4 - The probability of infection would provide a quantitative measure for measuring for the possibility of getting infection from contaminated multidose injectable product which is normally physically protected from environmental contamination with the exception of regularly penetrating syringe needle.

5 - Data calculation from Tables 3 (under the usual dosing system column) and 4 (in determination of microbial contamination density section) would allow for determination of the theoretical dose (D) of the microorganism for calculation of the response. The single spot contamination model assumed that infection occurred once with the second accidental use of the product with the same syringe. Equation of exponential doseresponse model of infection is as the following for Table 1 :

$$
\begin{gathered}
D_{4}=\left[\left(n /\left(V * f^{(4-1)}\right)\right]^{*} v\right. \\
P_{(\text {response) }}=1-\exp \left(-k * D_{4}\right)
\end{gathered}
$$

Where: $\mathrm{D}-$ dose of specific microbial particles to which patient was exposed in CFU; 4 - number of the dose rank during consumption of single unit dosage form; $\mathrm{n}$ - contamination level delivered to the product in vial through syringe needle (CFU); $\mathrm{V}-$ volume of the product during accidental contamination by $\mathrm{n}$ CFU $(\mathrm{ml}) ; f$ - reduction factor of microbial population in the pharmaceutical product obtained from the results of PET; $v-$ the common dose volume administered to the diabetic patients according to the product type as shown in Table $3 ; \mathrm{P}_{\text {(response) }}$ quantitative risk response of the disease response; $k$ - the microbial particle has Independent and identical probability of surviving to reach and infect at an appropriate site.

6 - For convinience of comparison between products the same potency of Insulin per day and the same logarithmic reduction (LR) were assumed for all types of formulae. Also the same contamination inoculum (CFU) of both Staphylococcus aureus and Pseudomonas aeruginosa was selected to contaminated different vial forms with the final count delivered by the needle was 7500 CFU.

\section{Statistical analysis}

Dose-response figure and models complex calculations of infections were designed using Microsoft Office Excel 2007. Nonparametric comparsion between probabilities of infections using Kruskal-Wallis test (One-Way ANOVA followed by Dunn's multiple comparisons test at $\alpha<0.05$ ) with the aid of GraphPad Prism v6 for windows. 
Table 3. Preservative efficacy test (PET) results of nine forms of recombinant human Insulin vials based on USP 51

\begin{tabular}{|c|c|c|c|c|c|}
\hline \multirow{2}{*}{ Human recombinant insulin type } & \multirow{2}{*}{ Test microorganisms* } & \multicolumn{3}{|c|}{ LR at testing days intervals } & \multirow{2}{*}{$\begin{array}{c}\text { Antimicrobial components / usual dosing } \\
\text { system }\end{array}$} \\
\hline & & 7 & 14 & 28 & \\
\hline \multirow[t]{5}{*}{ Highly purified isophane suspension (100 U-10 ml) vial } & S. aureus & $>5.66$ & $>5.66$ & $>5.66$ & \multirow{15}{*}{$\begin{array}{l}\text { Phenol and m-cresol } \\
50 \mathrm{U} \text { single dose/day }\end{array}$} \\
\hline & E. coli & $>5.64$ & $>5.64$ & $>5.64$ & \\
\hline & $P$. aeruginosa & $>5.46$ & $>5.46$ & $>5.46$ & \\
\hline & C. albicans & $>5.34$ & $>5.34$ & $>5.34$ & \\
\hline & A. brasiliensis & $>4.73$ & $>4.73$ & $>4.73$ & \\
\hline \multirow[t]{5}{*}{ Highly purified isophane suspension ( $100 \mathrm{U}-4 \mathrm{ml}$ ) vial } & S. aureus & $>5.83$ & $>5.83$ & $>5.83$ & \\
\hline & E. coli & $>5.72$ & $>5.72$ & $>5.72$ & \\
\hline & P. aeruginosa & $>5.29$ & $>5.29$ & $>5.29$ & \\
\hline & C. albicans & $>5.31$ & $>5.31$ & $>5.31$ & \\
\hline & A. brasiliensis & $>4.76$ & $>4.76$ & $>4.76$ & \\
\hline \multirow[t]{5}{*}{ Highly purified isophane suspension ( $40 \mathrm{U}-10 \mathrm{ml}$ ) vial } & S. aureus & $>5.34$ & $>5.34$ & $>5.34$ & \\
\hline & E. coli & $>5.19$ & $>5.19$ & $>5.19$ & \\
\hline & P. aeruginosa** & $>5.07$ & $>5.07$ & $>5.07$ & \\
\hline & C. albicans & $>4.62$ & $>4.62$ & $>4.62$ & \\
\hline & A. brasiliensis & $>4.31$ & $>4.31$ & $>4.31$ & \\
\hline \multirow[t]{5}{*}{ Highly purified regular solution (100 U-10 ml) vial } & S. aureus & $>5.63$ & $>5.63$ & $>5.63$ & \multirow{15}{*}{$50 \mathrm{U}$ divided in four doses/day } \\
\hline & E. coli & $>5.66$ & $>5.66$ & $>5.66$ & \\
\hline & P. aeruginosa & $>5.34$ & $>5.34$ & $>5.34$ & \\
\hline & C. albicans & $>5.29$ & $>5.29$ & $>5.29$ & \\
\hline & A. brasiliensis & $>4.71$ & $>4.71$ & $>4.71$ & \\
\hline \multirow[t]{5}{*}{ Highly purified regular solution (100 U-4 ml) vial } & S. aureus & $>5.70$ & $>5.70$ & $>5.70$ & \\
\hline & E. coli & $>5.53$ & $>5.53$ & $>5.53$ & \\
\hline & P. aeruginosa & $>5.28$ & $>5.28$ & $>5.28$ & \\
\hline & C. albicans & $>5.40$ & $>5.40$ & $>5.40$ & \\
\hline & A. brasiliensis & $>4.96$ & $>4.96$ & $>4.96$ & \\
\hline \multirow[t]{5}{*}{ Highly purified regular solution ( $40 \mathrm{U}-10 \mathrm{ml}$ ) vial } & S. aureus & $>5.34$ & $>5.34$ & $>5.34$ & \\
\hline & E. coli & $>5.15$ & $>5.15$ & $>5.15$ & \\
\hline & P. aeruginosa & $>5.07$ & $>5.07$ & $>5.07$ & \\
\hline & C. albicans & $>4.62$ & $>4.62$ & $>4.62$ & \\
\hline & A. brasiliensis & $>4.32$ & $>4.32$ & $>4.32$ & \\
\hline \multirow{5}{*}{$\begin{array}{l}\text { Highly purified regular } 30 \%+\text { isophane } 70 \% \text { (100 U-10 } \\
\text { ml) vial }\end{array}$} & S. aureus & $>5.37$ & $>5.37$ & $>5.37$ & \multirow{15}{*}{$\begin{array}{l}50 \mathrm{U} \text { divided two doses/day } \\
2 / 3+1 / 3\end{array}$} \\
\hline & E. coli & $>5.39$ & $>5.39$ & $>5.39$ & \\
\hline & P. aeruginosa & $>5.32$ & $>5.32$ & $>5.32$ & \\
\hline & C. albicans & $>5.07$ & $>5.07$ & $>5.07$ & \\
\hline & A. brasiliensis & $>4.47$ & $>4.47$ & $>4.47$ & \\
\hline \multirow{5}{*}{$\begin{array}{l}\text { Highly purified regular } 30 \%+\text { isophane } 70 \%(100 \mathrm{U}-4 \\
\mathrm{ml} \text { ) vial }\end{array}$} & S. aureus & $>5.48$ & $>5.48$ & $>5.48$ & \\
\hline & E. coli & $>5.51$ & $>5.51$ & $>5.51$ & \\
\hline & P. aeruginosa & $>5.54$ & $>5.54$ & $>5.54$ & \\
\hline & C. albicans & $>5.41$ & $>5.41$ & $>5.41$ & \\
\hline & A. brasiliensis & $>4.79$ & $>4.79$ & $>4.79$ & \\
\hline \multirow{5}{*}{$\begin{array}{l}\text { Highly purified regular } 30 \%+\text { isophane } 70 \% \text { (40 U-10 } \\
\text { ml) vial }\end{array}$} & S. aureus & $>5.48$ & $>5.48$ & $>5.48$ & \\
\hline & E. coli & $>5.58$ & $>5.58$ & $>5.58$ & \\
\hline & P. aeruginosa & $>5.27$ & $>5.27$ & $>5.27$ & \\
\hline & C. albicans & $>5.03$ & $>5.03$ & $>5.03$ & \\
\hline & A. brasiliensis & $>4.17$ & $>4.17$ & $>4.17$ & \\
\hline
\end{tabular}

$L R$, logarithimic reduction.

* All microorganisms have not been recovered at any stage of the test from the recovery medium.

** Selected cut-off value for the maximum risk of infection. 
Table 4. Parameters used in the assessement of the infection risk from contaminated products with indicator microbes

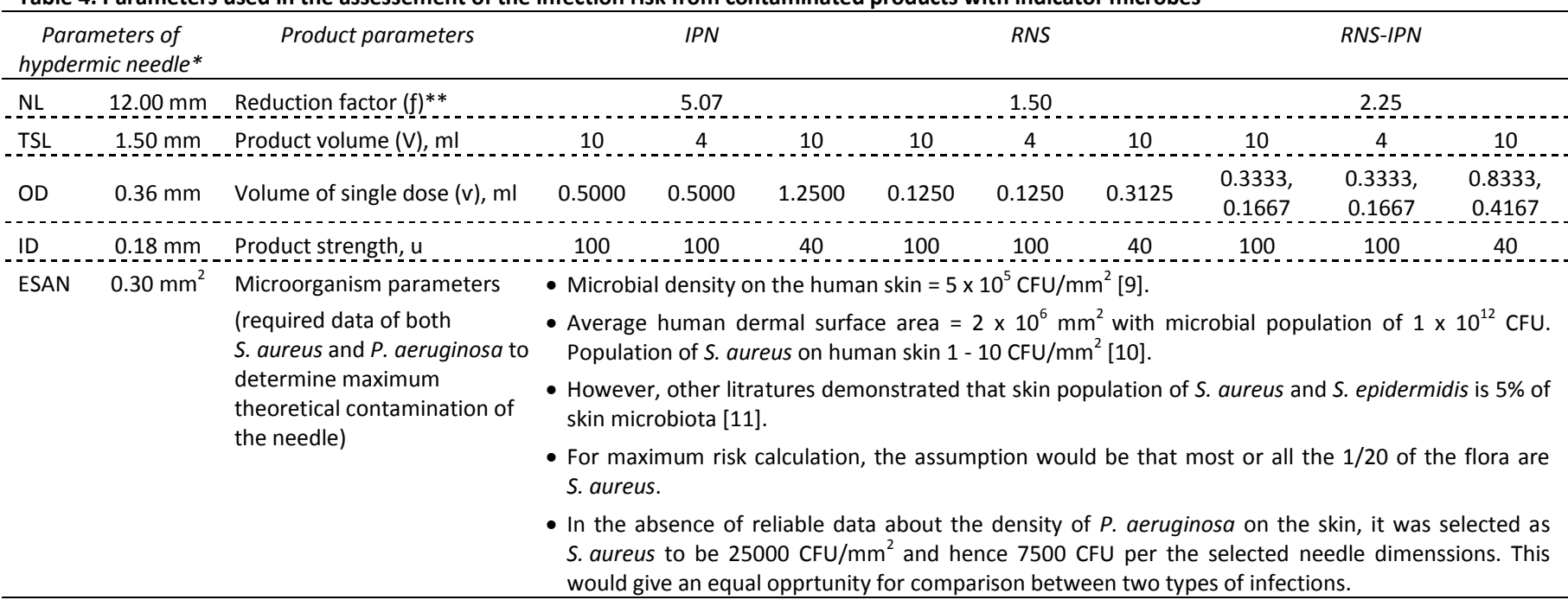

* Approximate measurement with digital caliber.

** Calculated from selected LR after 7 days of Table 3 from the lowest value in order to challenge the risk evaluation.

$\mathrm{NL}$, needle length; TSL, tip slope length; OD, outer diameter; ID, inner diameter; ESAN, exposed surface area of needle.
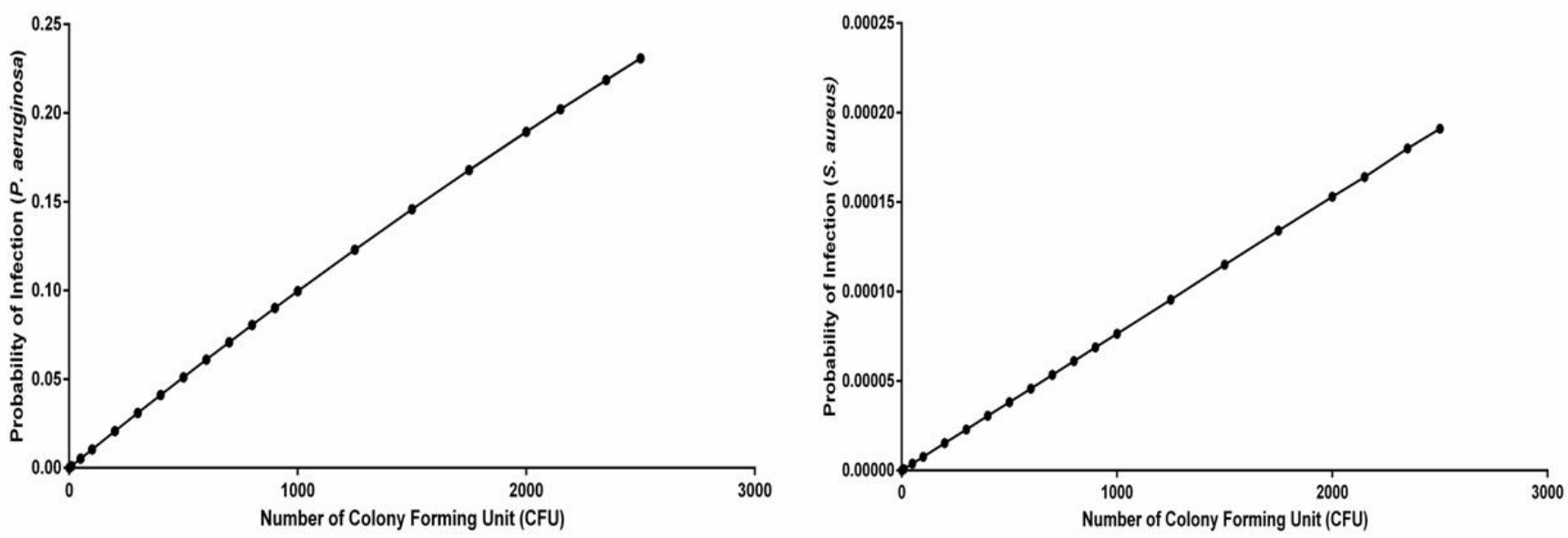

Figure 1. Exponetional relation of dose-response: model of infection fitted to $P$. aeruginosa (left Fig.) $y=0.00011010 * x^{0.9838960}\left(R^{2}=0.9998\right)$ and S. aureus (right Figure) $y=0.00000008 * x^{0.99998808}\left(R^{2}=1.0000\right)$ based on estimated population intruding the products through contaminated hypodermic syring needle.

\section{Results and Discussion}

AET results are demonstrated in Table 3 which exceeded the acceptance criteria of the test by achieving overkill ability in eradicating pharmacopial microbes with notably no recovery of colonies in culture plates after seven, 14 and 28 days for each type of Insulin product. Hence, the lowest cut-off value of bacteria of 5.07 (about 1.07 reduction factor within hour) was used of assessing microbial reduction in the contaminated product. Neutralization procedure is a crucial preliminary test to ensure the validity of PET. The applied neutralization technique based on pharmacopeial guide was dilution method according to the principle of concentration exponent $(\eta)$. Phenol $(\eta=6)$ ten to 100 folds dilution reduce activity by $1 \times 10^{6}$ to $1 \times 10^{12}$ times. Phenolic compounds $(\eta=4-9.9)$ ten to 100 folds dilution reduce activity by times $1 \times 10^{4}-1 \times 10^{8}$ to $7.9 \times 10^{9}-6.3 \times 10^{19}$ [8]. Hence, the dilution technique in buffer or saline was applied as convinient method of stopping antimicrobial activity when testing AET. Primary data that was required for assessing the infection risks are shown in Table 4. Table 4 demonstrated the surface area of the penetrating part of the needle with the exposed surface area calculated (using data of microbial density to estimate the theoretical microbial density on the needle surface) in addition to the usual dosing system was determined. By determining the starting contamination level to be 7500 CFU from the table (Figure 1), could be obtained which demonstrated the relation between the number of infecting particles and the probability of infection from both type of the reference microorganisms. By assuming the maximum risk by starting contamination with the second administration with the same syringe, the product would be exposed to 7500 CFU theoretically. If this practice of misusing the syringe was not repeated then the washout periods with the associated risks would be as demonstrated in Table 5. 
Table 5. Probability of infection from single-spot contamination of nine forms Insulin vial products using $S$. aureus and $P$. aeruginosa as model microorganisms Successive doses of administration till risk of infection falls below 1:1000000*

Insulin type
Highly purified isophane suspension (100 U-10 ml) vial

Highly purified isophane

suspension (100 U-4 ml) vial

Highly purified isophane suspension $(40 \mathrm{U}-10 \mathrm{ml})$ vial

Highly purified regular solution

(100 U-10 ml) vial

$$
\begin{gathered}
\text { Successive doses of ad } \\
\text { S. aureus (subcutaneous) }
\end{gathered}
$$

0.0060, 0.0006, 0.0001 (3 administered doses)

$0.0164,0.0016,0.0003,0.0001$ (4 administered doses)

$0.0066,0.0016,0.0003,0.0001$ (4 administered doses)

$0.0060,0.0005,0.0003,0.0002,0.0001,0.0001,0.0001(7$

$0.0060,0.0005,0.0003,0.0002,0.0001,0.0001,0.0001(7$ administered doses)

Highly purified regular solution

(100 U-4 ml) vial
$0.0148,0.0012,0.0008,0.0005,0.0004,0.0002,0.0002$

$0.0001,0.0001$, (9 administered doses)
$P$. aeruginosa (septecemia)

$7.9603,0.8147,0.1612,0.0318,0.0063,0.0012,0.0002$ (7 administered doses)

$20.1496,2.1946,0.4367,0.0863,0.0170,0.0034,0.0007$ (7 administered doses)

$8.6151,2.1967,0.4371,0.0864,0.0170,0.0034,0.0007$, 0.0001 ( 8 administered doses)

$7.8636,0.6802,0.4540,0.3029,0.2020,0.1347,0.0898$ $0.0599,0.0399,0.0266,0.0178,0.0118,0.0079,0.0053$ $0.0035,0.0023,0.0016,0.0010,0.0007,0.0005,0.0003$, $0.0002,0.0001,0.0001,0.0001$ (25 administered doses) $18.3950,1.6797,1.1230,0.7501,0.5007,0.3341,0.2228$, $0.1486,0.0991,0.0661,0.0441,0.0294,0.0196,0.0131$ $0.0087,0.0058,0.0039,0.0026,0.0017,0.0011,0.0008$, $0.0005,0.0003,0.0002,0.0002,0.0001,0.0001$ (27 administered doses)
Highly purified regular solution (40 U-10 ml) vial

$0.0059,0.0012,0.0008,0.0005,0.0004,0.0002,0.0002$, $0.0001,0.0001$ ( 9 administered doses)

$7.8152,1.6810,1.1238,0.7506,0.5011,0.3343,0.2230$, $0.1487,0.0992,0.0661,0.0441,0.0294,0.0196,0.0131$ $0.0087,0.0058,0.0039,0.0026,0.0017,0.0011,0.0008$ $0.0005,0.0003,0.0002,0.0002,0.0001,0.0001$ (27 administered doses)

Highly purified regular $30 \%+\quad 0.0059,0.0004,0.0004,0.0001,0.0001$ (5 administered isophane $70 \%$ (100 U-10 ml) vial doses)

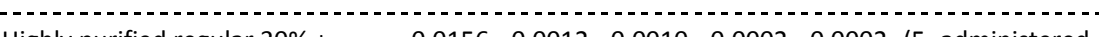

Highly purified regular $30 \%+$ isophane $70 \%(100 \mathrm{U}-4 \mathrm{ml})$ vial

Highly purified regular $30 \%+$

isophane $70 \%$ (40 U-10 $\mathrm{ml}$ ) vial

$0.0156,0.0012,0.0010,0.0002,0.0002$ (5 administered doses)

$0.0063,0.0012,0.0010,0.0002,0.0002(5$ administered doses)
$7.8249,0.6019,0.5345,0.1192,0.1058,0.0236,0.0209$ $0.0047,0.0041,0.0009,0.0008,0.0002,0.0002 \quad(13$ administered doses)

$19.3321,1.5791,1.4032,0.3139,0.2787,0.0621,0.0551$ (7 administered doses)**

$8.2401,1.5800,1.4050,0.3141,0.2791,0.0621,0.0552$ $0.0123,0.0109,0.0024,0.0022,0.0005,0.0004,0.0001$, 0.0001 ( 15 administered doses)

\footnotetext{
* The values of probability of infections are actually lower than tabulated results based on the cut-off figure of PET number of $>1.07$ reduction folds per hour because there was no microorganisms recovered in the AET.

** Infection risk persists at probability 551:1000000 level with the final dose consumption from the pharmaceutical unit.

N.B. The decreasing risk of microbial infection (from initially contaminated product which originated from the reuse of contaminated needle with the second application and use) was determined until either the product vial is used up (i.e. empty) or the risk value has declined till reaching value less 1 per million, which of the nearest.
}

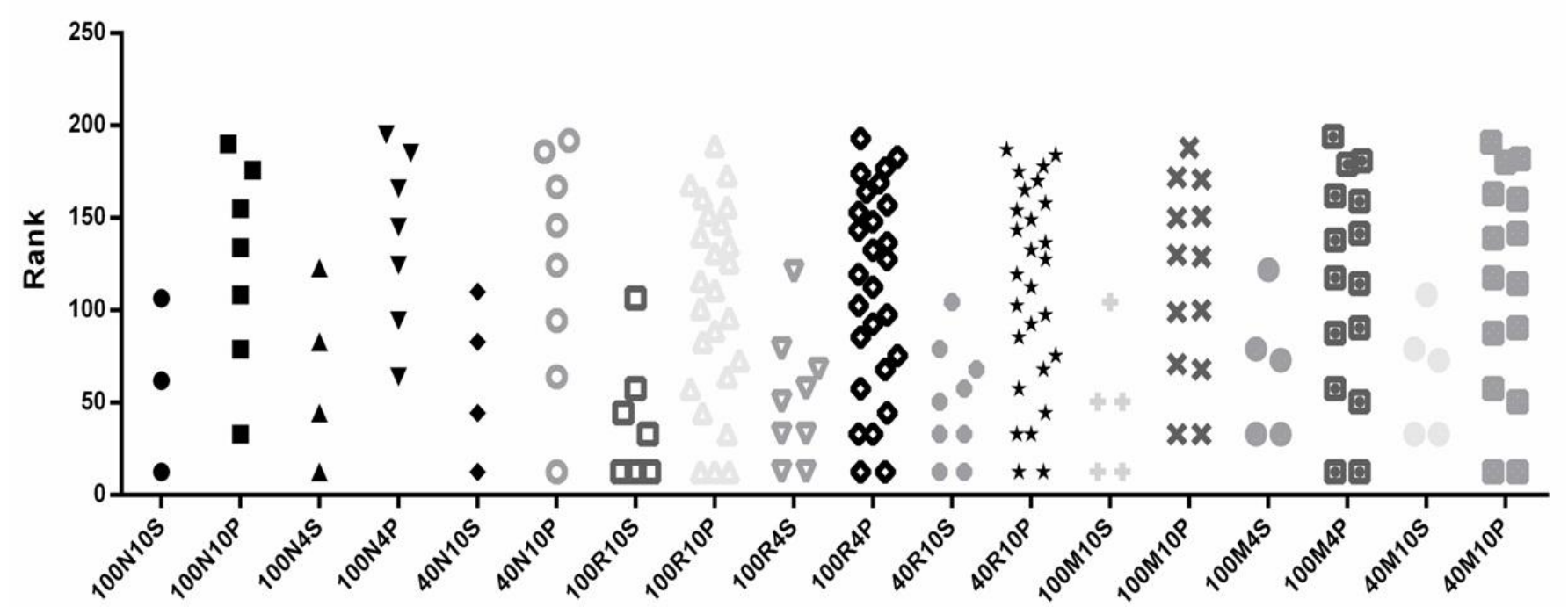

Figure 2: Kruskal-Wallis test: One-Way ANOVA followed by Dunn's multiple comparisons test at $P<0.05$ of probabilities of infections expressed as percents. Key of $x$ axis: Number $(100$ or $40 \mathrm{U}) /$ Letter $(\mathrm{N}, \mathrm{R}$ or $\mathrm{M}) /$ Number $(10$ or $4 \mathrm{ml}) /$ Letter $(\mathrm{S}$. aureus $(\mathrm{S})$ or $P$. aeruginosa $(\mathrm{P}))=$ Potency $(100$ or $40 \mathrm{U}) / \mathrm{Insulin}$ type/ Volume (10 or $4 \mathrm{ml}) /$ Microorganism model $(\mathrm{P}=$ Pseudomonas aeruginosa or $\mathrm{S}=$ Staphylococcus aureus $)$. 
It should be noted that the risk of infection is still relatively high at one or two doses following initial contamination, which then ceases very rapdily and then steadily decline shallowly. Statistical analysis between groups was demonstrated in (Figure 2) using Kruskal-Wallis test. Also, from Table 5 the geometric average of the total risk for each product could be calculated and they were $0.0349 \%, 0.0936 \%, 0.0370 \%, 0.0086 \%, 0.0140 \%, 0.0263 \%$, $0.0165 \%, 0.0189 \%$ and $0.0179 \%$ for $P$. aeruginosa while they were $0.0007 \%, 0.0009 \%, 0.0007 \%, 0.0003 \%, 0.0005 \%, 0.0004 \%$, $0.0004 \%, 0.0010 \%$ and $0.0008 \%$ for $S$. aureus, with the same order sequence of products in Table 4 . The means were largely dependent on the number of doses and the extent of tailing in the profile of microbial infection probability.

Considerations in selection of the indicator microorganisms can be clearly demonstrated by the fact that Staphylococci are very common skin flora with notably $S$. aureus being known as potenial opportunistic pathogen [9] and has well studied doseresponse model of infection [12]. On the other hand, $P$. aeruginosa is considered one of the most common microbes that infects blood stream and participate by more than $10 \%$ of the Gram-negative hospital and community-aquired septicemia 1997 and the rate of infections is $0.064 \%[13,14]$. It has also a model of infection that suites septicemia situations [12].

\section{Conclusions and Future Directions}

The current approach provided quantitative method for assessing the risk possibility of infection from contaminated multidose Insulin product. The finding of this simulation study would provide insight for modeling other methods of infection not only for injectable products but also for different forms of medicinal products including oral liquid and topical drugs. While the present case study has maximized the value of using AET, yet it highlighted clearly the limitations of the standard method. PET applied the overkilling principle at relatively long intervals which is not suitable for the actual in-use situations in which contamination with low number bioburden is most likely especially in the current case in which the product is physically isolated from the environment and the access to the contents is gained through very limited area (syringe needle). Moreover, administration dosing intervals and volumes plays an important rules for the magnitude and extent of contamination transfer and hence the possibility of infection. The current quantitative risk method applied provided a new insight and approach that can be used as milestone for experts in the pharmaceutical industry to customize not only the product formula, but also all influential parameters that impact the health and safety of patients.

\section{Acknowledgments}

This work was supported partially financially by HIKMA Pharma pharmaceutical company $-2^{\text {nd }}$ Industrial zone $-6^{\text {th }}$ of October city. The practical part of all experiments was performed in the microbiology laboratory in the quality control department. Thanks to Mr. Ahmed Saber Nouby for supplying technical assistance with work in PET. Data gathering and issuing was performed by HIKMA microbiology laboratory team. Reference and writing style review was performed by Dr. Engy Refaat Rashed.

\section{Conflict of interest}

The author declares that he has no conflict of interest.

\section{References}

1. Denyer SP. Microbial contamination of intravenous fluids during use. Br J Pharm Practice 1984; 6: 122-126.

2. Arduino MJ, Bland LA, McAllister SK, Aguero SM, Villarino ME, McNeil $\mathrm{MM}$, et al. Microbial growth and endotoxin production in the intravenous anesthetic propofol. Infect Control Hosp Epidemiol 1991; 12(9): 535-539. (PMID: 1940276)

3. Koerner RJ, Morgan S, Ford M, Orr KE, McComb JM, Gould FK. Outbreak of Gram-negative septicaemia caused by contaminated continuous infusions prepared in a non-clinical area. J Hosp Infect 1997; 36(4): 285-289. (PMID: 9261758)

4. USP 36-NF31, Chapter 51. Antimicrobial effectiveness testing. United States Pharmacopoeia 36/National Formulary 31, Baltimore, MD, USA 2014.

5. Eissa ME, Mahmoud AM. Assessment of modified preservation formulae after optimizing preliminary neutralization evaluation study. International Journal of Multidisciplinary Research Studies and Developments 2015; 1(2): 5-11.

6. Rose JB, Haas CN. A risk assessment framework for the evaluation of skin infections and the potential impact of antibacterial soap washing. Am J Infect Control 1999; 27(6): S26-S33. (doi: 10.1016/S01966553(99)70039-8) (PMID: 10586143)

7. Hazlett LD, Rosen DD, Berk RS. Age-related susceptibility to Pseudomonas aeruginosa ocular infections in mice. Infection and immunity 1978; 20(1): 25-29. (PMID: 669795)

8. USP 36-NF31, Chapter 1227. Validation of microbial recovery from pharmacopeial articles. United States Pharmacopoeia 36/National Formulary 31, Baltimore, MD, USA, 2014.

9. Online Textbook of Bacteriology [Internet]. Textbookofbacteriology.net. 2006 [cited 15 March 2015]. Available from: http://www.textbookofbacteriology.net/

10. Kowalski W. Hospital airborne infection control. Boca Raton, Fla.: CRC Press, 2012.

11. Grice EA, Kong HH, Renaud G, Young AC, Bouffard GG, Blakesley RW, et al. A diversity profile of the human skin microbiota. Genome Research 2008; 18(7): 1043-1050. (doi: 10.1101/gr.075549.107) (PMID: 18502944)

12. Dose response assessment - QMRAwiki [Internet]. Qmrawiki.canr.msu.edu. 2016 [cited 13 April 2015]. Available from: http://qmrawiki.canr.msu.edu/index.php?title=Dose_response_assess ment

13. Diekema DJ, Pfaller MA, Jones RN, Doern GV, Winokur PL, Gales AC, et al. Survey of bloodstream infections due to gram-negative bacilli: frequency of occurrence and antimicrobial susceptibility of isolates collected in the United States, Canada, and Latin America for the SENTRY Antimicrobial Surveillance Program, 1997. Clin Inf Dis 1999; 29(3): 595-607. (doi: 10.1086/598640) (PMID: 10530454)

14. Al-Hasan MN, Wilson JW, Lahr BD, Eckel-Passow JE, Baddour LM Incidence of Pseudomonas aeruginosa bacteremia: a population-based study. Am J Med 2008; 121(8): 702-708. (PMID: 18691484) (doi: 10.1016/j.amjmed.2008.03.029)

\section{Authors:}

Mostafa Essam Eissa - MSc, Quality Control Section Head, Microbiology Laboratory Division, HIKMA Pharma Pharmaceutical Company, Giza, Egypt. 